\title{
Regulation of the histone acetyltransferase activity of hMOF via autoacetylation of Lys 274
}

Cell Research (2011) 21:1262-1266. doi:10.1038/cr.2011.105; published online 21 June 2011

\section{Dear Editor,}

Males-absent-on-the-first (MOF, also called MYST1 or KAT8) is a histone acetyltransferase (HAT) belonging to the MOZ, Ybf2/Sas3, Sas2 and Tip60 (MYST) family. MOF has been shown to possess a specific HAT activity towards Lys 16 of histone H4 (H4K16) [1]. Homozygous knockout of MOF in mice results in loss of H4K16 acetylation and embryonic lethality, indicating that MOF and H4K16 acetylation are essential for embryogenesis and genome stability in mammals [2]. Downregulation of human MOF (hMOF) leads to dramatic nuclear morphological deformation and inhibition of cell cycle progression [3], and has recently been correlated with primary breast carcinoma and medulloblastoma [4].

Here we report the crystal structure of the catalytic domain (residues 174-449) of hMOF at $2.1 \AA$ A resolution (Figure 1 and Supplementary information, Data S1, Figure S1A and Table S1). Intriguingly, in the initial difference Fourier maps there was strong residual electron density at the tip of the side chain of Lys274 at the catalytic active site (Figure 1A, left panel). Modeling of the density as an acetyl group and further refinement of the acetylated Lys274 led to a good fit of the map (Figure $1 \mathrm{~A}$, right panel). Structural comparison indicates that the conformation of the catalytic domain of the apo hMOF is similar to that of hMOF in complex with acetylcoenzyme A (acetyl-CoA) alone (PDB code 2GIV) and in complex with acetyl-CoA and an male-specific lethal 1 (MSL1) fragment [5]. In particular, the acetylation of Lys274 is found in all these structures. The acetylation of Lys274 in the purified hMOF protein (hereafter the hMOF protein will refer to the catalytic domain of hMOF unless otherwise specified) was verified with liquid chromatography-tandem mass spectrometry (LC-MS/ MS) (Supplementary information, Figure S1B and S1C). Although other acetylation sites were also identified, the intensity of the peptide containing the acetylated Lys 274 constituted $83 \%$ of the total intensity of the acetylated peptides, indicating that Lys274 is the major acetylation site (Supplementary information, Table S2). Further- more, hMOF is also acetylated at Lys274 in vivo (see discussion later). Sequence alignment of hMOF with other members of the MYST family HATs reveals that among all the identified acetylation sites, Lys274 is the only strictly conserved residue, suggesting that acetylation of this site might be common and probably functionally important for the MYST family HATs. Lys274 and two other strictly conserved residues (Cys316 and Glu350) which are in the vicinity of the acetyl group of the cofactor acetyl-CoA were mutated to study their functional roles (Supplementary information, Data S1 and Figure S2). Strikingly, mutation of Lys274 to Arg (K274R) almost completely abolished the hMOF activity $(0.1 \%)$, while the K274Q mutant retained about $2.9 \%$ activity (Supplementary information, Figure S2A), suggesting that the loss of the HAT activity by the K274R mutation could be due to the inability of Arg to be acetylated.

To investigate whether Lys 274 is autoacetylated, the wild-type and mutant hMOF proteins were incubated with acetyl-CoA and subsequently subjected to western blot with an antibody specific to acetylated lysine. Intriguingly, for all the hMOF proteins except the K274Q mutant, the acetylation levels prior to the acetyl-CoA incubation and the extents of the increase after the incubation correlated well with their respective HAT activities (Supplementary information, Figure S2), indicating that the acetylation of hMOF is catalyzed by hMOF itself. Although the K274Q mutant had an HAT activity comparable to that of the G327E mutant, its acetylation was undetected, which is in agreement with our MS data that Lys 274 is the main acetylation site of the hMOF catalytic domain.

Next we investigated whether the autoacetylation of Lys274 of hMOF occurs inter- or intra-molecularly. The GST-tagged hMOF was incubated with either His-tagged wild-type or mutant hMOF in the presence or absence of acetyl-CoA (Figure 1B). Both GST-tagged and Histagged wild-type hMOF could autoacetylate themselves; however, the His-tagged K274R and G329E mutants exhibited no or very weak activity. The presence of any of the His-tagged hMOF proteins had no appreciable effect 
A
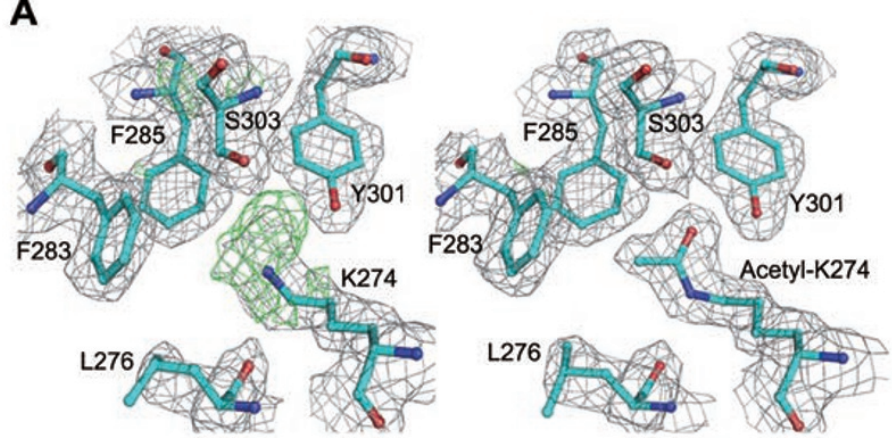

B

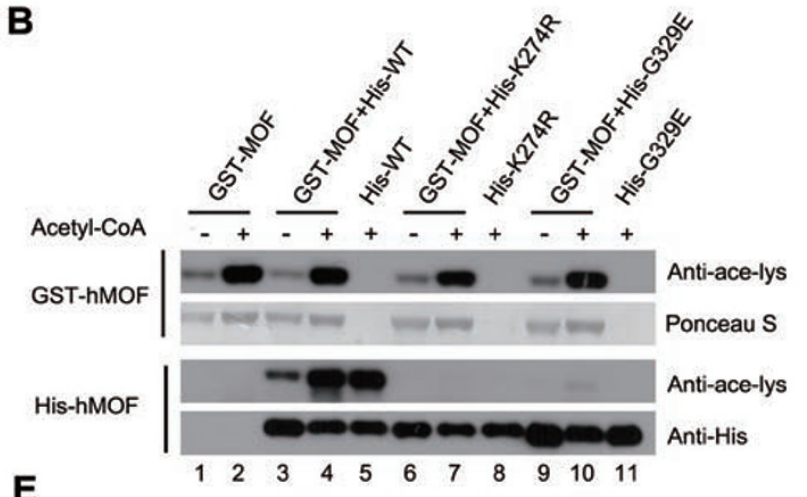

E

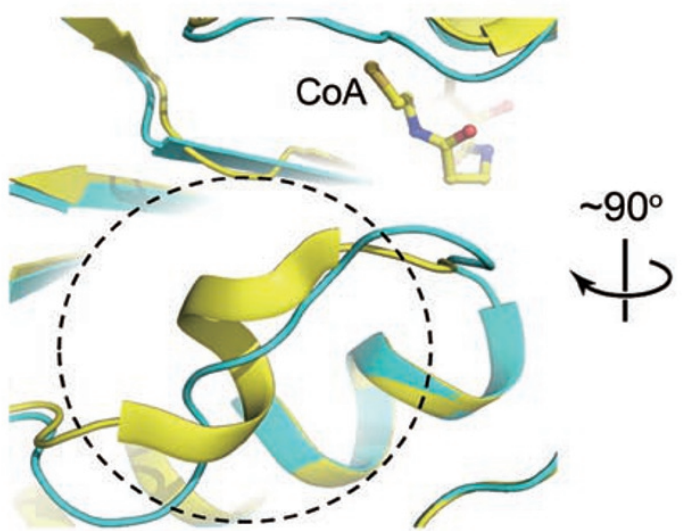

$\mathbf{F}$

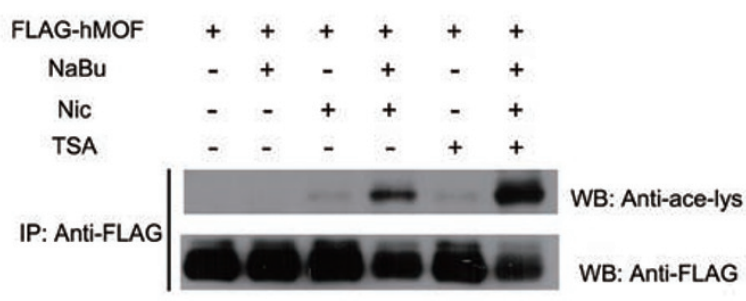

C

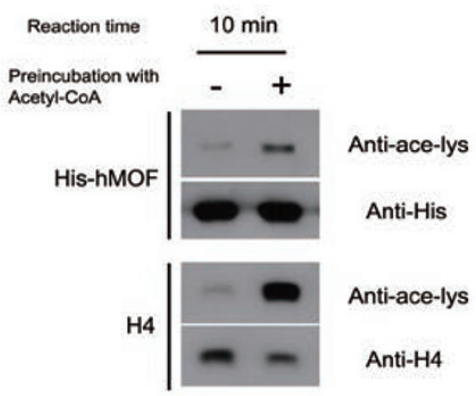

D

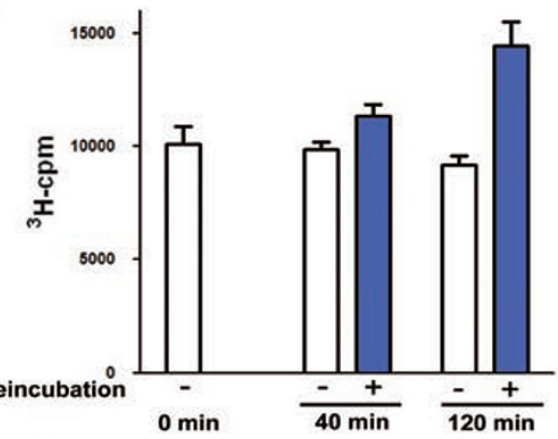

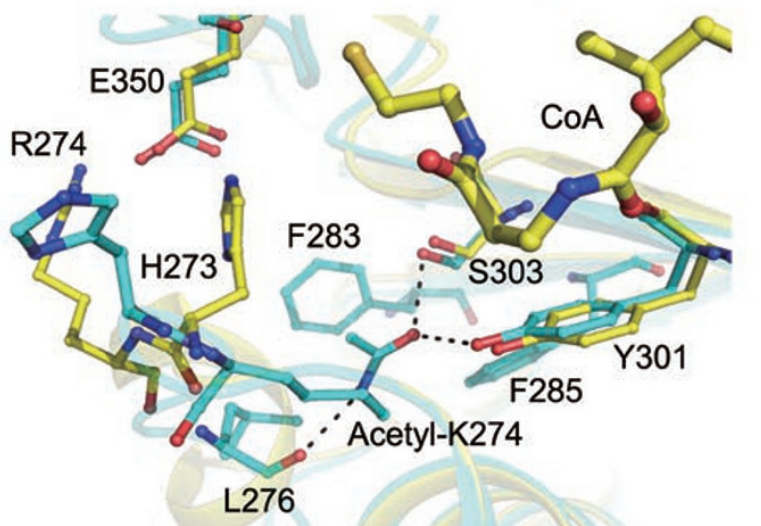

G

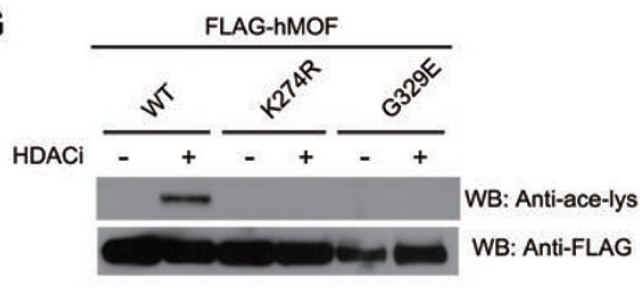

IP: Anti-FLAG on the acetylation level of the GST-tagged hMOF and vice versa. These results indicate that Lys 274 of hMOF is autoacetylated in an intra-molecular manner.

To investigate whether acetylation of this residue is functionally important, the HAT activity of hMOF with or without pre-incubation with low concentration of acetyl-CoA $(200 \mu \mathrm{M})$ was examined with the HAT activity assays in the presence of high concentration of acetyl-CoA $(1 \mathrm{mM})$. As shown in Figure 1C, hMOF preincubated with acetyl-CoA can acetylate H4 more efficiently. To exclude the possibility that pre-incubation of hMOF with acetyl-CoA allows binding of acetyl-CoA in a higher percentage of the enzyme hence leading to higher activity, we carried out more quantitative HAT activity 
Figure 1 Regulation of the histone acetyltransferase activity of hMOF via autoacetylation of Lys274. (A) A close-up view of $2 F_{\mathrm{o}}-F_{\mathrm{c}}$ and $F_{\mathrm{o}}-F_{\mathrm{c}}$ maps at Lys 274 and surrounding residues. The $2 F_{\mathrm{o}}-F_{\mathrm{c}}$ (gray, $1 \sigma$ ) and $F_{\mathrm{o}}-F_{\mathrm{c}}$ (green, $2.5 \sigma$ ) maps are shown after refinement of the side chain of Lys274 as unmodified (left) or acetylated (right). (B) Intra-molecular autoacetylation of hMOF. Approximately $10 \mu \mathrm{g}$ of GST-tagged and $5 \mu \mathrm{g}$ of His-tagged hMOF proteins were incubated with $200 \mu \mathrm{M}$ acetyl-CoA $(+)$ or the mock solution (-) for $2.5 \mathrm{~h}$ at $30^{\circ} \mathrm{C}$. The acetylation level of the proteins was examined. (C) Stimulation of the HAT activity of hMOF by Lys 274 autoacetylation. Approximately $0.8 \mu \mathrm{g}$ of hMOF proteins with (+) or without (-) pre-incubation with $200 \mu \mathrm{M}$ acetyl-CoA were subjected to HAT activity assays. (D) Quantitative HAT activity assays of autoacetylated hMOF. Approximately $1 \mu \mathrm{M}$ hMOF protein was pre-incubated with $25 \mu \mathrm{M}$ non-labeled acetyl-CoA (+) or the mock solution (-) for 40 or $120 \mathrm{~min}$ at $30{ }^{\circ} \mathrm{C}$. HAT activity assays were carried out in the presence of $25 \mu \mathrm{M}{ }^{3} \mathrm{H}$-labeled acetyl-CoA with $50 \mu \mathrm{M}$ recombinant $\mathrm{H} 4$ as substrate for $10 \mathrm{~min}$. The activities were detected with liquid scintillation. (E) Comparison of the active site of the wild-type hMOF (cyan) with that of the K274R mutant (PDB code 2PQ8, yellow). Left: the major structural difference of the $\alpha 3-\beta 7$ segment (residues 272-278) (marked with a circle). Right: A detailed view of the differed region and nearby residues. The hydrogen-bonding interactions between the side chain of acetylated Lys274 and the surrounding residues are indicated with dashed lines. (F) Enhanced acetylation of the full-length hMOF with the treatment of HDAC inhibitors. 293T cells transfected with FLAG-hMOF were treated with $10 \mathrm{mM}$ sodium butyrate ( $\mathrm{NaBu}$, an inhibitor of Class I and Class II HDACs except for HDAC6 and HDAC10), $1 \mathrm{mM}$ nicotinamide (Nic, an inhibitor of the Sirtin family HDACs), and/or $5 \mu \mathrm{M}$ trichostatin A (TSA, an inhibitor of Class I and Class II HDACs) for 6 h. (G) Autoacetylation of hMOF in 293T cells. 293T cells transfected with the plasmids encoding the full-length wild-type, K274R or G329E mutant hMOF were treated with (+) or without (-) a combination of the three HDAC inhibitors (HDACi). For (F and $\mathbf{G}$ ), the FLAG-hMOF proteins were immunoprecipitated with anti-FLAG M2 beads and then assayed with western blot using anti-ace-lys and anti-FLAG antibodies.

assays with addition of non-labeled acetyl-CoA at the pre-incubation step and supplementation of ${ }^{3} \mathrm{H}$-labeled acetyl-CoA at the activity assay step. The results show that pre-incubation with acetyl-CoA increases the hMOF activity in a time-dependent manner (Figure 1D), indicating that autoacetylation of hMOF potentiates its HAT activity. It has been reported that moderate modulation of the activities of HATs could have a significant impact on the biological processes in which they are involved. For example, an approximate $25 \%$ increase in the enzymatic activity of TIP60, which is most closely related to hMOF in the MYST family, by the binding of PDCD5 led to acceleration of DNA damage-induced apoptosis [6]. Therefore, the increase in the enzymatic activity of hMOF we observed in Figure 1C and 1D is not trivial and suggests an important role for acetylation of Lys 274 in the HAT activity of hMOF.

The structures of the wild-type hMOF (PDB code 2 GIV and in this study) were compared with the structure of the K274R mutant (PDB code 2PQ8) which might mimic the structure of hMOF with an unacetylated Lys274. Although the overall structures are similar, the region encompassing residues $272-278$ (the $\alpha 3-\beta 7$ segment) exhibits significant conformational difference which forms a loop in the wild-type hMOF but a $33_{10}$-helix in the K274R mutant (Figure 1E). Within this segment, the side chains of acetylated Lys274 and Arg274 point to opposite sides although their main chains occupy similar positions.

In the K274R mutant structure, the side chain of Arg274 flips outwards from the active site and forms a salt bridge with the carboxyl group of Glu350 (2.7 $\AA$ )
(Supplementary information, Table S3). Consequently, the side chain of His273 occupies the space between Glu350 and cofactor coenzyme A (CoA) (Figure 1E, right panel). Superposition of this structure onto that of Tetrahymena Gen5 bound with CoA and a histone H3 peptide [7] shows that the side chain of hMOF His 273 occupies a site corresponding to that between the highly conserved Glu and the cofactor in the Gen5 structure which is the binding site for the substrate lysine of the H3 peptide (Supplementary information, Figure S3). Therefore, the complete loss of the HAT activity of hMOF by the K274R mutation might be due to impaired binding and/or improper positioning of the side chain of the substrate lysine, and hence the active site of the K274R mutant hMOF represents an inactive conformation.

In contrast, in the wild-type hMOF, the side chain of acetylated Lys274 points inwards forming part of the active site (Figure 1E). Correspondingly, His273 occupies a position different from that in the mutant structure and the space between Glu350 and cofactor CoA is vacant, allowing for proper binding and positioning of the substrate lysine (Figure 1E, right panel). A detailed analysis shows that Lys274 makes extensive interactions with the surrounding residues. The acetyl carbonyl group forms two hydrogen bonds with the side chains of two conserved residues Tyr301 and Ser303. The acetyl methyl group makes hydrophobic interactions with two aromatic residues Phe283 and Phe285. In addition, the $\varepsilon$-nitrogen of Lys274 forms a hydrogen bond with the main-chain carbonyl group of Leu276 (Figure 1E, right panel). Besides these interactions via the side chain of acetylated 
Lys274, there are only three additional hydrogen bonds between the $\alpha 3-\beta 7$ segment and the surrounding residues (Supplementary information, Table S4). Therefore, the interactions made by the acetylated side chain of Lys 274 constitute a major part of the interaction network which helps maintain the loop conformation of the $\alpha 3-\beta 7$ segment and the corresponding structure of the active site.

The loop conformation of the $\alpha 3-\beta 7$ segment might also exist in solution, as in the wild-type hMOF structures no residue in this region was involved in crystal packing and this conformation was captured independently under different crystallization conditions [5]. Thus, the active site of the wild-type hMOF structures is likely to represent a biologically active conformation. On the other hand, as lysine shares a similar electrostatic property as arginine, hMOF with an unacetylated Lys 274 might assume an inactive conformation of the catalytic site similar to that of the K274R mutant which, intriguingly, might block the binding of MSL1 due to the steric hindrance between the main-chain carbonyl group of hMOF-Tyr277 and the side chain of MSL1-Glu516 (Supplementary information, Figure S4). Therefore, acetylation of Lys274 may play a critical role for the enzymatic activity through stabilization of the loop conformation of the $\alpha 3-\beta 7$ segment and hence the active conformation of the catalytic active site.

The effect of Lys274 acetylation of hMOF on its HAT activity was further investigated in vivo. Acetylation was detected for the full-length hMOF overexpressed in 293T cells although the acetylation level was low (Supplementary information, Figure S5A). Treatment of the cells with $10 \mathrm{mM}$ sodium butyrate, $1 \mathrm{mM}$ nicotinamide or 5 $\mu \mathrm{M}$ TSA alone had no or minor effect on the acetylation level of the full-length hMOF; however, treatment with a combination of sodium butyrate and nicotinamide or the three inhibitors resulted in significant increase of the hMOF acetylation level (Figure 1F), indicating that multiple classes of histone deacetylases (HDACs) are likely responsible for the deacetylation of hMOF. In contrast, no acetylation was detected for the K274R and G329E mutants even when the cells were treated with the HDAC inhibitors (Figure 1G). Consistently, LC-MS/MS analysis of the full-length hMOF immunoprecipitated from 293T cells treated with the three HDAC inhibitors confirmed that Lys274 is acetylated in vivo (Supplementary information, Figure S5B). The potential biological function of Lys 274 acetylation of hMOF was further investigated by examination of the H4K16 acetylation level in COS7 cells. Overexpression of the wild-type hMOF resulted in higher level of H4K16 acetylation compared with the control, while the K274R mutant functions as a dominant negative form (Supplementary information, Data S1 and
Figure S5C), supporting the notion that Lys274 and probably its acetylation are critical for the hMOF function and the regulation of H4K16 acetylation level in vivo.

Recently the Akhtar and Liu groups also report that hMOF can be autoacetylated at Lys274 [5, 8]. Compared with their studies, our work reveals that hMOF is autoacetylated in an intra-molecular manner, indicates that the autoacetylation of hMOF at Lys274 has a significant impact on the catalytic activity of the enzyme, and provides the structural basis for the modulation of the enzymatic activity by Lys 274 acetylation. Thus, our study investigates independently the function of this modification from different aspects. It was shown that the acetyltransferase p300/CBP can be autoacetylated at multiple lysine residues on a pseudo substrate inhibitory loop leading to substantial increase of the HAT activity [9]. Another HAT, Rtt109 was found to be autoacetylated at Lys 290 of the activation domain, which is essential for its HAT activity towards H3K56 [10]. Lys274 of hMOF and Lys290 of Rtt109 are not at equivalent positions (Supplementary information, Figure S6), and the regulatory scheme of hMOF by Lys 274 acetylation proposed in this study is quite different from those of p300 and Rtt109. Therefore, hMOF employs a novel regulatory mechanism of acetyltransferase activities, providing another example of great divergence in the regulation of acetyltransferases as well as other enzymes by acetylation.

The structure of the hMOF catalytic domain has been deposited with the RCSB Protein Data Bank under accession code $3 \mathrm{QAH}$.

\section{Acknowledgments}

We thank the staff members at SSRF, China for support in diffraction data collection. This work was supported by MOST (2011CB911102 and 2011CB966301), the National Natural Science Foundation of China (30730028), CAS (SIBS2008002, KSCX2-EW-Q-1-03), and STCSM (10JC1416500).

\section{Bingfa Sun ${ }^{1,4}$, Shunling Guo ${ }^{3,4}$, Qingyu Tang ${ }^{1,4}$, Chen $\mathrm{Li}^{2}$, Rong Zeng ${ }^{2}$, Zhiqi Xiong ${ }^{3}$, Chen Zhong ${ }^{1}$, Jianping Ding ${ }^{1}$}

${ }^{I}$ State Key Laboratory of Molecular Biology and Research Center for Structural Biology, ${ }^{2}$ Key Laboratory of Systems Biology, Institute of Biochemistry and Cell Biology, ${ }^{3}$ State Key Laboratory of Neuroscience, Institute of Neuroscience, Shanghai Institutes for Biological Sciences, Chinese Academy of Sciences, ${ }^{4}$ Graduate School of Chinese Academy of Sciences, 320 Yue Yang Road, Shanghai 200031, China

Correspondence: Jianping Ding

Tel: +86-21-54921619, Fax: +86-21-5492-1116

E-mail: jpding@sibs.ac.cn 


\section{References}

1 Akhtar A, Becker PB. Activation of transcription through histone $\mathrm{H} 4$ acetylation by MOF, an acetyltransferase essential for dosage compensation in Drosophila. Mol Cell 2000; 5:367-375.

2 Thomas T, Dixon MP, Kueh AJ, Voss AK. Mof (MYST1 or KAT8) is essential for progression of embryonic development past the blastocyst stage and required for normal chromatin architecture. Mol Cell Biol 2008; 28:5093-5105.

3 Taipale M, Rea S, Richter K, et al. hMOF histone acetyltransferase is required for histone $\mathrm{H} 4$ lysine 16 acetylation in mammalian cells. Mol Cell Biol 2005; 25:6798-6810.

4 Pfister S, Rea S, Taipale M, et al. The histone acetyltransferase hMOF is frequently downregulated in primary breast carcinoma and medulloblastoma and constitutes a biomarker for clinical outcome in medulloblastoma. Int J Cancer 2008; 122:1207-1213.

5 Kadlec J, Hallacli E, Lipp M, et al. Structural basis for MOF and MSL3 recruitment into the dosage compensation complex by MSL1. Nat Struct Mol Biol 2011; 18:142-149.

6 Xu L, Chen Y, Song Q, Xu D, Wang Y, Ma D. PDCD5 interacts with Tip60 and functions as a cooperator in acetyltransferase activity and DNA damage-induced apoptosis. Neoplasia 2009; 11:345-354.

7 Rojas JR, Trievel RC, Zhou J, et al. Structure of Tetrahymena GCN5 bound to coenzyme A and a histone H3 peptide. Nature 1999; 401:93-98.

8 Lu L, Li L, Lv X, Wu XS, Liu DP, Liang CC. Modulations of hMOF autoacetylation by SIRT1 regulate hMOF recruitment and activities on the chromatin. Cell Res 2011; 21:1182-1195.

9 Thompson PR, Wang D, Wang L, et al. Regulation of the p300 HAT domain via a novel activation loop. Nat Struct Mol Biol 2004; 11:308-315.

10 Stavropoulos P, Nagy V, Blobel G, Hoelz A. Molecular basis for the autoregulation of the protein acetyl transferase Rtt109. Proc Natl Acad Sci USA 2008; 105:12236-12241.

(Supplementary information is linked to the online version of the paper on the Cell Research website.) 